\title{
Spanwise Domain Effects on the Evolution of the Plane Turbulent Mixing Layer
}

\author{
W. A. McMullan ${ }^{\mathrm{a} *}$ \\ ${ }^{a}$ Department of Engineering, University of Leicester, University Road, Leicester, LE1 7RH;
}

(Received 00 Month 20XX; final version received 00 Month 20XX)

\begin{abstract}
Large Eddy Simulation is used to simulate a series of plane mixing layers. The influence of the spanwise domain on the development of the mixing layer, and the evolution of the coherent structures are considered. The mixing layers originate from laminar conditions, and an idealised inflow condition is found to produce accurate flow predictions when the spanwise computational domain extent is sufficient to avoid confinement effects. Spanwise domain confinement of the flow occurs when the ratio of spanwise domain extent to local momentum thickness reaches a value of ten. Flow confinement results in changes to both the growth mechanism of the turbulent coherent structures, and the nature of the interactions that occur between them. The results demonstrate that simulations of the two-dimensional mixing layer flow requires a three-dimensional computational domain in order that the flow will evolve in a manner that is free from restraints imposed by the spanwise domain.
\end{abstract}

Keywords: Mixing Layer, Large Eddy Simulation, Coherent Structures, Flow Confinement, Vortices

\section{Introduction}

The plane mixing layer is commonly viewed as a canonical flow type, and its simple geometry lends itself favourably to both experimental and numerical research. Experimental studies over the past 70 years have shown, however, that the mixing layer is in fact a very complicated flow configuration. For apparently identical initial conditions, the growth rate of the single stream shear layer can vary by as much as $30 \%$ between experimental facilities (Liepmann and Laufer 1947; Wygnanski and Fiedler 1970). It was later shown that this discrepancy may have been caused by the state of the separating boundary layer (Batt 1975), with a turbulent boundary layer producing a shear layer which grows more quickly than a flow developing from a laminar boundary layer. In two-stream mixing layers, however, the converse appears to be true; an initially-turbulent high-speed boundary layer produces a mixing layer with a lower growth rate than one which develops from an initiallylaminar high-speed boundary layer (Karasso and Mungal 1996; Slessor, Bond and Dimotakis 1998).

A further complication in the study of the mixing layer is the presence of quasi-two-dimensional coherent structures embedded in the turbulent flow, first discovered by Brown and Roshko (1974). Since their discovery, coherent structures in the plane mixing layer have been the subject of extensive research. In spite of the large body of published research on the mixing layer, the mechanism of growth and entrainment in the flow has yet to be fully described. Experiments on mixing layers at modest Reynolds numbers have shown that the mixing layer grows through successive interactions between primary spanwise Kelvin-Helmholtz (K-H) vortices (Winant and Browand 1974). Analysis of flow visualisations of high Reynolds number mixing layer suggests that a significant proportion of the growth of the mixing layer occurs between interactions (Hernan and Jimenez 1982). Recent experimental work by D'Ovidio and Coats (2013) suggests that both mechanisms

*Corresponding author. Email: andrew.mcmullan@le.ac.uk 
exist in the mixing layer; in the pre-transition region the mixing layer grows through pairing-type interactions, whilst in the post-transition region the self-similar growth of the mixing layer is accounted for by the continuous linear growth of the coherent structures (D'Ovidio and Coats 2013). The distinction between the laminar and turbulent regions of the flow is marked by the mixing transition (Konrad 1976). The transition occurs with the interaction of primary spanwise vortices, but also requires the presence of secondary, streamwise vortices (Huang and Ho 1990). In plane turbulent mixing layers, the mixing transition occurs once the local Reynolds number, based on the velocity difference across the layer and its visual thickness is approximately 10,000 (Dimotakis 2000).

The development and evolution of streamwise vorticity in the mixing layer has been the study of extensive research. The streamwise vortex structure was observed to manifest itself as a 'streaky' structure in schlieren images (Konrad 1976). Experimental evidence suggests that, for a given set of initial conditions, the secondary structure appears to form at fixed spanwise locations (Huang and Ho 1990; Bell and Mehta 1992; Bernal and Roshko 1986) and persist far into the fully developed self-similar region of the mixing layer (Bell and Mehta 1992). Extensive experimental work has been undertaken to ascertain the origin of streamwise vorticity in the mixing layer. It has been shown that the position of the streaks across the span can change, depending on the upstream flow conditions (Bernal and Roshko 1986; Jimenez 1983; Lasheras et al. 1986). Theoretical analysis has shown that streamwise vorticity can be formed through helical pairings, or via a translative instability (Pierrehumbert and Widnall 1982).

Numerical simulations offer the potential to study mixing layers in a controlled environment, where the effect of initial conditions can be assessed accurately. However, there is a significant computational cost involved in simulating the fully time-dependent flow and many early numerical studies of the mixing layer focused on the temporally-evolving flow, where the periodicity of the flow substantially reduces the computational cost of the simulations. Temporal mixing layer simulations have predicted many of the features observed in the real flow (Moser and Rogers 1991; Rogers and Moser 1992; Moser and Rogers 1993), but they are unable to predict the high-speed stream entrainment bias observed in experiments. Spatially developing mixing layer simulations require a large computational domain, as well as the specification of inflow and outflow boundary conditions. It is common to impose hyperbolic tangent inflow conditions (Wang, Tanahashi and Miyauchi 2007; Attili and Bisetti 2012) but other research has shown that this type of inflow condition significantly affects the growth and entrainment of the flow (McMullan, Gao and Coats 2007, 2009; Soteriou and Ghoniem 1998; Soteriou and Yang 1999). The imposition of boundary layer profiles, which include the wake deficit from the presence of the splitter plate, produces flow characteristics in much better agreement with experiment (McMullan, Gao and Coats 2007; Soteriou and Ghoniem 1998).

As the mixing layer is recognised as a statistically two-dimensional flow, it is common practice to perform spatially-developing mixing layer simulations in a flow confined to a two-dimensional box, or in a computational domain with a narrow spanwise domain extent (Yang et al. 2004; Zhou and Pereira 2000; Biancofore 2014). For flows confined to two-dimensional boxes, the mixing layer does not undergo a transition to turbulence, as the lack of spanwise domain prohibits the formation of secondary, streamwise structure (McMullan, Gao and Coats 2010). A recent study has shown that a narrow three-dimensional computational domain restricts the growth of the mixing layer, and a criterion for the minimum computational domain size was determined (Biancofore 2014). It is not clear, however, how the imposed spanwise domain extent affects the evolution of the coherent structures in the flow, and it should be noted that the study of Biancofore (2014) did not compare results with a reference experiment. The secondary streamwise structure observed in numerical simulations of the plane mixing layer appears to be a function of the imposed inflow conditions. Simulations with three-dimensional white-noise fluctuations produce oblique rollers that undergo pre-transition localised pairing interactions or helical pairings (Lesieur et al. 1997; Bernard 2008), with the pre-transition vortex structure taking the appearance of a chain-link fence. In contrast, highly two-dimensional disturbances produce thin rib vortices in the interconnecting braid region 
between primary rollers (Lesieur et al. 1997).

The aim of this research is to quantify the effects of the spanwise computational domain extent on the coherent structures present in the spatially-developing, initially-laminar mixing layer. The imposition of a finite spanwise extent will limit the maximum spanwise length-scale possible in the flow, even with the imposition of spanwise periodic boundary conditions. The experimental conditions of Browand and Latigo (1979) are simulated using Large Eddy Simulation (LES). Three computational domains of varying spanwise extent are simulated, and the influence of the spanwise domain on the flow statistics is assessed. Flow visualisation images are used to interrogate the structures in the simulations, and the effect of the spanwise extent on the evolution of the coherent structures is highlighted. A criterion for the minimum spanwise domain required for an unconfined flow simulation is then postulated. To assist with flow visualisation, a density difference of less than $0.04 \%$ is imposed between the freestreams - this permits the use of numerical schlieren visualisation techniques for what is essentially a uniform-density mixing layer (McMullan, Coats and Gao 2011).

Details of the reference experiment are outlined in Section 2. The parameters and setup of the simulations reported here are outlined in Section 3. An overview of the numerical algorithms that are used in the code is given in Section 4. Simulation results and discussion are provided in Section 5 , and concluding remarks are drawn in Section 6.

\section{Reference Experiment}

The experiments of Browand and Latigo (1979) provide the flow conditions that are replicated by LES in this study. The large test section used in the experiments permitted a long streamwise extent of the flow to be measured. The upper and lower walls of the facility were fixed, which resulted in a small adverse pressure gradient in the flow. The adverse pressure gradient produced a decreasing low-speed side freestream velocity with increasing streamwise distance from the splitter plate, and as such the measured mixing layer may not be considered as truly self-preserving. The experimental conditions originating from initially laminar conditions are simulated in the present study, and the flow parameters are given in Table 1 . The velocity ratio parameter, $R$, is defined as

$$
R=\frac{U_{1}-U_{2}}{U_{1}+U_{2}}
$$

and has a value of $R=0.66$ at the trailing edge of the splitter plate.

The dataset of Browand and Latigo is attractive for numerical simulation as the initial conditions of the mixing layer are reasonably well documented. The laminar boundary layer profiles for both streams are reported, along with the variation of the root mean squared (r.m.s.) streamwise velocity fluctuation. Several useful flow statistics are also documented, which permits careful assessment of numerical simulation data against the reference experiment.

\section{Simulation Parameters}

In this research, three computational domains are used to assess the effect of the imposed spanwise domain on the evolution of the simulated mixing layer. A summary of the computational domains is given in Table 2. Each domain extends $1630 \theta_{i} \times 1326 \theta_{i}$ in the streamwise $(x)$, and vertical (y) directions respectively, where $\theta_{i}$ is the initial momentum thickness of the flow. The initial momentum thickness is equivalent to the momentum thickness of the high-speed boundary layer as it departs from the splitter plate. The spanwise $(z)$ domain extent varies from $L_{z} / \theta_{i}=98$ in case D1, successively doubling between cases up to $L_{z} / \theta_{i}=392$ in case D3. The domain begins at the trailing edge of the splitter plate, and as such there is no solid geometry contained within the computational domain. 
To permit valid comparisons between each simulation, the mesh resolution is held constant between all three cases. The mesh resolution used in this study is derived from previous simulations (McMullan, Gao and Coats 2009, 2015). In those studies, the mesh resolution was validated against both reference DNS and experimental data. It was shown that a mesh of the current resolution produces satisfactory flow statistics when compared to Direct Numerical Simulation, and that the mesh can also capture the large-scale coherent structures present in the turbulent mixing layer. The streamwise and cross-stream domain directions are discretised into $768 \times 256$ cells respectively. The grid is refined in the plane of the splitter plate, resulting in minimum grid spacings of $\Delta x_{\min } / \theta_{i}=0.46$, and $\Delta y_{\min } / \theta_{i}=0.086$. The high-speed laminar boundary layer is resolved with 36 points in the cross-stream direction. The grid is progressively stretched in the streamwise and cross-stream directions to permit a reduced cell count in regions of low flow variability far from the plane of the splitter plate. The grid spacing in the spanwise direction is uniform, and held constant for all three cases. In the largest computational domain presented here (D3), there are a total of 50.3 million cells in the computational domain. A validation study performed elsewhere (McMullan, Gao and Coats 2015) has shown that large scale evolution of the flow is insensitive to the choice of WALE model parameter on a mesh of comparable resolution. In that study, WALE model coefficients in the range of $C_{w}=0.3-0.56$ were tested, and in the present study the WALE model coefficient is given a value of $C_{w}=0.56$ for all runs.

The non-dimensional time step of each simulation is set to $\Delta t /\left(\theta_{i} / U_{c}\right)=0.0205$, where $U_{c}=$ $\left(U_{1}+U_{2}\right) / 2$ is the convection velocity of the flow. These flows were simulated over $1.2 \mathrm{million}$ time steps, with the initial 260,000 time steps required to produce a statistically stationary flow field. Flow visualisation images were then recorded at a sampling rate of $1.667 \mathrm{kHz}$, and statistical samples were recorded at every time step during the remainder of the run. The total number of statistical samples is ameliorated through the spanwise averaging of the statistics. Care is taken to ensure that spanwise averaging over different spanwise domain extents in each simulation does not skew the mean-flow statistics - the convergence of each set of statistics is checked to satisfy a convergence criterion. The maximum reported CFL number in the simulations was 0.3. In order to visualise the flow field using numerical schlieren, there is a small difference in density between the two freestreams. The density ratio is set to $s=\rho_{2} / \rho_{1}=0.9996$, and hence the flow can essentially be considered as being of uniform density. As described in Section 4 the density field is linked to the transport of a passive scalar. The passive scalar has a value of unity in the high-speed stream, and zero in the low-speed stream.

As the boundary layers in the experiment were laminar in nature, the inflow condition for each simulation is prescribed through the use of Blasius laminar boundary layer profiles. The momentum thickness of each profile matches the experimental data given in Table 1. Pseudo-random white noise fluctuations are superposed onto the inflow profile in order to allow the natural selection of the dominant instability modes of the flow. The magnitude of the imposed fluctuations replicates those recorded in the experiment. This type of white noise disturbance environment has been used in other simulation studies of the plane mixing layer, with good mixing layer statistical properties obtained (Wang, Tanahashi and Miyauchi 2007; Attili and Bisetti 2012; McMullan, Gao and Coats 2015). The upper and lower walls of the computational domain are modelled as slip walls. The spanwise boundaries are periodic in nature. A standard advective boundary condition is employed at the outflow plane of the domain. These simulations, with simple inflow boundary conditions, represent an idealised form of the experiment.

\section{Code overview}

The research code is based on the low-Mach number approximation of the spatially-filtered governing equations. The low-Mach number approximation permits the simulation of variable density flows in an incompressible computational framework. This is attractive in the present study as it prevents the simulation time step from being limited by the speed of sound, as sound waves are 
eliminated from the flow-field. The filtered low-Mach number continuity, momentum and scalar transport governing equations can be written as

$$
\begin{gathered}
\frac{\partial \bar{\rho}}{\partial t}+\frac{\partial \bar{\rho} \tilde{u}_{i}}{\partial x_{i}}=0 \\
\frac{\partial \bar{\rho} \tilde{u}_{i}}{\partial t}+\frac{\partial \bar{\rho} \tilde{u}_{i} \tilde{u}_{j}}{\partial x_{j}}=-\frac{\partial \bar{p}}{\partial x_{i}}+\frac{\partial \tau_{i j}}{\partial x_{j}}-\frac{\partial T_{i j}}{\partial x_{j}} \\
\frac{\partial \bar{\rho} \tilde{\xi}}{\partial t}+\frac{\partial \bar{\rho} \tilde{u}_{i} \tilde{\xi}}{\partial x_{i}}=\frac{\partial J_{i}}{\partial x_{i}}-\frac{\partial M_{i}}{\partial x_{i}}
\end{gathered}
$$

where a quantity $\bar{\phi}$ denotes a spatially-filtered variable, and $\tilde{\phi}$ denotes a Favre-averaged variable. For a Newtonian fluid obeying Fick's law of diffusion, the mass flux is given by $J_{i}=\gamma \frac{\partial \tilde{\xi}}{\partial x_{i}}$, where $\gamma=\bar{\rho} \Gamma$ denotes the mass diffusivity coefficient. The filtering operation introduces extra terms into the governing equations which must be modelled to close the system. The subgrid stress and mass flux tensors are given by $T_{i j}=\bar{\rho}\left(\widetilde{u_{i} u_{j}}-\tilde{u_{i}} \tilde{u}_{j}\right)$ and $M_{i}=\bar{\rho}\left(\widetilde{u_{i} \xi}-\tilde{u_{i}} \tilde{\xi}\right)$.

The variables are discretised using the finite volume method in a staggered-cell arrangement. Second-order central-differencing schemes are used for the convection and diffusion terms in the solution of the momentum equation, and a flux-limited, second-order accurate upwinding scheme is used for scalar fluxes (Gao and Voke 1995). This choice of scheme for the scalar terms minimises out of bounds errors in the calculations of the passive scalar. Time advancement is achieved through the second-order accurate Adams-Bashforth method. The pressure equation is solved using a multigrid method. The outflow condition applied in the simulations is a convective condition similar to that used in many previous studies (Voke and Potamitis 1994), and has been shown to be passive.

A subgrid-scale model must be used to close the momentum equation, and the code employs the WALE model, (Nicoud and Ducros 1999) given by

$$
\mu_{\text {sgs }}=\bar{\rho}\left(C_{w} \Delta\right)^{2} \frac{\left(S_{i j}^{d} S_{i j}^{d}\right)^{3 / 2}}{\left(\bar{S}_{i j} \bar{S}_{i j}\right)^{5 / 2}+\left(S_{i j}^{d} S_{i j}^{d}\right)^{5 / 4}}
$$

where $S_{i j}^{d}=\frac{1}{2}\left(\bar{g}_{i j}^{2}+\bar{g}_{j i}^{2}\right)-\frac{1}{3} \delta_{i j} \bar{g}_{k k}^{2}, \bar{g}_{i j}=\partial \tilde{u}_{i} / \partial x_{j}$, and $C_{w}$ is a model constant specified $a$ priori. The WALE model is attractive for the simulation of free shear flows with initially laminar conditions, as it predicts zero eddy viscosity in the presence of pure shear. It has been shown in other work that this model produces improved plane mixing layer predictions when compared to the standard Smagorinsky model (McMullan, Gao and Coats 2015).

The mass fraction transport equation is related to the density field (Wang et al. 2008) and is closed with the commonly-used gradient-diffusion model. A constant turbulent Schmidt number is assumed in this model, such that

$$
M_{i}=-\frac{\mu_{s g s}}{S c_{t}}\left(\frac{\partial \tilde{\xi}}{\partial x_{i}}\right)
$$

In this study the subgrid-scale Schmidt number is set to $S c_{t}=0.3$. 


\section{Results}

The simulation results are normalised in the same manner as the experiments. Where velocity statistics are normalised by $\Delta U=U_{1}-U_{2}$, the local values of streamwise velocity are used.

\subsection{Mean Flow Statistics}

A commonly-used measure of the integral thickness of the mixing layer is the momentum thickness, $\theta$. This quantity is defined as

$$
\theta=\frac{1}{\Delta U^{2}} \int_{-\infty}^{\infty}\left(U_{1}-\bar{u}_{t}\right)\left(\bar{u}_{t}-U_{2}\right) d y
$$

where $\bar{u}_{t}$ is the mean streamwise velocity. The variation of the normalised momentum thickness with streamwise distance is plotted in Figure 1a for all simulations. The downwards curving of the momentum thickness near to the outflow boundary is common in simulations of plane mixing layers (Wang, Tanahashi and Miyauchi 2007; Attili and Bisetti 2012; Biancofore 2014; McMullan, Gao and Coats 2015), and is caused by the interruption of the entrainment of fluid into the coherent structures which are passing through the outflow plane.

Of the simulations presented here, it is Case D3 which produces the best comparison with experiment, with a linear gradient in the self-similar region up to the point where the outflow boundary influences the growth of the coherent structures in the flow. The evolution of the momentum thickness in the range of $0 \leq x / \theta_{i} \leq 360$ is the same for all three simulations, and downstream of $x \approx$ $360 \theta_{i}$ the momentum thickness of Case D1 diverges from that of the other two. A linear gradient in the momentum thickness is obtained at $x \approx 400 \theta_{i}$. Case D2 has the same momentum thickness evolution as Case D3 in the range of $0 \leq x / \theta_{i} \leq 760$, downstream of which the growth of the mixing layer is less rapid.

The aspect ratio of the computational domain, $A$, is defined as the ratio of the spanwise domain length to the local momentum thickness of the mixing layer, $A=L_{z} / \theta$. The variation of the aspect ratio with streamwise distance is shown in Figure 1b. It is interesting to note that in Case D1 at $x \approx 360 \theta_{i}$, and in Case D2 at $x \approx 760 \theta_{i}$ the aspect ratio of the domain is $A \approx 10$. These locations correspond to where the momentum thickness of each simulation diverges from that of Case D3. This shows that the mixing layer evolution becomes adversely affected by the spanwise domain extent when the aspect ratio reaches a value of ten. The aspect ratio of Case D3 does not attain a value of ten within the computational domain, and therefore maintains a uniform rate of growth in the self-similar region of the mixing layer.

The momentum thickness growth rate of the flow, $d \theta / d x$, can be obtained from the self-similar region of the momentum thickness curves where the gradient is linear. This quantity is presented in Table 3 for each case. Simulation D2 has two growth rates tabulated, corresponding to the two distinct linear growth regions observed in Figure 1. For a self-preserving, uniform density mixing layer, the growth rate of the momentum thickness is expected to vary as

$$
\frac{d \theta}{d x}=k_{1} R
$$

where $k_{1}$ is a constant. The value of $k_{1}$ for Case D3 falls within the range of mixing layer thickness growth rate constants reported in the literature (Brown and Roshko 1974; Bernard 2008). When the flow development becomes inhibited by the spanwise domain extent in both cases D1 and D2, the momentum thickness growth rate decreases.

An alternative measure of the spreading of the mixing layer is the visual thickness. This is de- 
termined by superimposing many instantaneous numerical schlieren visualisations and tracing the wedge swept out by the mixing layer, often referred to as the $1 \%$ thickness. This is consistent with an analogous method used in previous experimental studies where flow visualisations were investigated, (Karasso and Mungal 1996; Brown and Roshko 1974) and previous numerical simulations. (McMullan, Coats and Gao 2011) In addition to determining the mean visual thickness of the flow the virtual origin, $x_{0}$, is also acquired using this method. The visual thickness growth rate is expected to follow the relationship

$$
\delta_{v i z}^{\prime}=\frac{\delta_{v i z}}{x-x_{0}}=k_{2} R
$$

where $k_{2}$ is a constant. The visual thickness growth rates are given in Table 3 . It is known that the visual thickness is a factor of ten larger than the momentum thickness for a uniform density flow, and as such $k_{2} \approx 10 k_{1}$ should be valid for the present simulations. The data in Table 3 shows that only Case D3 produces growth rate constants $k_{1}$ and $k_{2}$ which maintain this proportionality. For Case D1, $k_{2}=11.67 k_{1}$ - this discrepancy when compared to available experimental evidence suggests that this simulations is not a physically realistic representation of the plane turbulent mixing layer flow found in the reference experiment. Case D2 demonstrates the correct proportionality of the growth rates up to $x / \theta_{i}=760$.

The normalised mean streamwise velocity of the flow at $x / \theta_{i}=1000$ is shown in Figure $2 \mathrm{a}$ for all cases. There is extremely good agreement between simulation and experimental data for all cases. Profiles of streamwise root mean squared velocity fluctuations recorded at the same streamwise location are shown in Figure 2b. In the experimental data the measurements were made with a hot-wire probe, and the data is the resultant velocity in the $x-y$ plane. To within experimental error, Cases D2 and D3 produce accurate predictions of this quantity. In Case D1, the velocity fluctuations are over-predicted towards the outer edge of the mixing layer in both streams, and the peak fluctuation is slightly under-predicted. The evolution of the maximum r.m.s. streamwise velocity fluctuation with downstream distance is plotted in Figure 3. All simulations show a large over-prediction of the peak magnitude of the fluctuation at $x / \theta_{i} \approx 250$. This type of over-prediction is common in numerical simulations of shear layers developing from initially laminar conditions.(McMullan, Gao and Coats 2015; Bogey and Bailly 2010). In the self-similar region of the flow it is Case D3 which, to within experimental error, produces the best comparison with the reference data. The data of Case D1 diverges from the prediction of Case D3 at a streamwise location of $x / \theta_{i} \approx 360$, and that of Case D2 diverges at $x / \theta_{i} \approx 760$. These two streamwise locations are similar to the streamwise locations where the aspect ratio of the flow attains a value of $A=10$. Prior to the spanwise wavelength of the mixing layer being inhibited by the size of the spanwise domain, there are no obvious artefacts in Cases D1 and D2 from the presence of the spanwise periodicity in the flow. Figure 3 demonstrates that, once the spanwise wavelength of the mixing layer has become confined by the spanwise domain, the streamwise velocity fluctuation level in the mixing level decreases compared to that of an unconfined flow.

\subsection{Flow Visualisation}

The statistical data reported above demonstrates some significant differences in the three simulations reported here. These statistical differences are caused by underlying changes in the flow evolution between the simulations. To quantify these differences, instantaneous flow visualisation images are interrogated. The time-dependent nature of LES permits the tracking of individual structures in the computational domain, and can offer insights into the growth and entrainment mechanisms of the mixing layer (McMullan, Gao and Coats 2009, 2015).

The small density difference between the freestreams permits the use of numerical schlieren flow visualisation. In the current research, numerical schlieren is computed from the divergence of the 
density field, with the visualisation images rendered using the scaling proposed by Quirk (1994) and Hadjadj and Kudryavstev (2005). In experimental flow visualisation, schlieren and shadowgraph techniques produce images that are effectively averaged representations of the flow along the direction of interrogation of the optical beam. To maintain consistency with the experimental visualisation techniques, the $x-y$ images presented here (referred to as 'side-view') are averaged across the span of the domain, and the $x-z$ images (referred to as 'plan-view') are averaged along the cross-stream direction.

Representative instantaneous flow visualisation outputs from Cases D1 and D3 are shown in Figures 4 and 5 respectively. The outputs share common features; there are obvious spanwise vortex-like structures in the side-view, the size and spacing of which increase with downstream distance in the mixing layer. In these particular images, the region of $0 \leq x / \theta_{i} \leq 360$ is occupied by pre-transition Kelvin-Helmholtz vortices. In the plan view, the initial $\mathrm{K}-\mathrm{H}$ vortex which forms from the roll-up of the mixing layer extends across the entire span. A secondary instability then forms in the interconnecting braid region between the primary structures, which is visible as streamwiseorientated structures in the plan view. Representative $y-z$ cuts of the passive scalar distribution through a pre-transition vortex core at $x / \theta_{i}=108$ are shown in Figures $6 \mathrm{a}-6 \mathrm{c}$ for each of the three cases presented here. Instantaneous velocity vectors are also shown on the images. The formation of the secondary streamwise structure is manifest through a long-wavelength undulation across the span of the primary vortex structure. The wavelength of this secondary instability is qualitatively similar in each case. This is not surprising because none of the simulations are adversely affected by the spanwise domain extent at this streamwise location.

Typical $Q$-criterion (Haller 2005) iso-surfaces in the pre-transition region of each simulation are shown in Figure 7. All simulations show that the pre-transition primary vortices undergo localised interactions, which are particularly evident in Case D1 (Figure 7a) at $x / \theta_{i} \approx 220$ and in Case D3 at $x / \theta_{i} \approx 180$. The vortex structure visualised in these images is remarkably similar to that observed in other simulations of the pre-transition region of the mixing layer (Bernard 2008; Lesieur et al. 1997), where the pre-transition structure takes a 'chain-link fence' appearance. There is no particular evidence for a 'streaky' streamwise structure which has been observed in experimental studies (Bernal and Roshko 1986; Bell and Mehta 1992), and has also been observed to co-exist with the chain-link fence structure in numerical simulations (Bernard 2008).

Once the streamwise vortex structure is present in the mixing layer the next amalgamative interaction that occurs between primary vortices precipitates the transition to turbulence. All simulations predict a mean transition location of $x_{t} \approx 330 \theta_{i}$, which corresponds to a pairing parameter value (Huang and Ho 1990) of $x^{*}=7.26$. This value is roughly in the region of the mean location of the second vortex interaction. The mean local Reynolds number at which the transition occurs, based on velocity difference across the layer and its visual thickness is $R e_{\delta} \approx$ 40,000, exceeding the postulated criterion of $10^{4}$ (Dimotakis 2000). Beyond the mixing transition, the spanwise coherent structure evident in the side-view image has a complex three-dimensional structure embedded within it in the plan view image. In the plan view of Figure 4 (Case D1), it can be qualitatively observed that the scale of the streamwise structure beyond $x / \theta_{i} \approx 550$ is comparable to that of the spanwise domain. In Case D3, however, the scale of the streamwise structures is significantly smaller than the spanwise domain extent, permitting a row of streamwise vortices to be wrapped around the primary structure. Further evidence of this arrangement can be seen in Figure 8, where instantaneous $y-z$ images of the passive scalar distribution in the core of a coherent structure is presented for Cases D1 and D3. These images were recorded at a streamwise location of $x / \theta_{i}=1304$, far into the fully-developed region of the mixing layer. In Case D1, the scalar distribution displays evidence of two rows of 'mushroom-shaped' eruptions, on the upper and lower side of the mixing layer respectively. These scalar patterns indicate the presence of streamwise vorticity in the flow. The scale of this streamwise structure, however, is very large with respect to the spanwise domain, and at most one wavelength of structure can be observed. In Case D3, in contrast, there are several eruptions distributed across the span on both sides of the mixing layer, highlighted in the image by the broken circles. 
These flow visualisation images indicate that the effect of spanwise confinement is to prevent the secondary structure from evolving beyond a maximum size, determined by the spanwise domain extent. Even with the imposition of spanwise periodic boundary conditions, the maximum spanwise wavelength permissible in the flow is dictated by the extent of the spanwise domain. This effect has been observed in Large Eddy Simulations of compressor blade flows (McMullan and Page 2011). The influence that this effect has on the evolution of the coherent structures in the mixing layer is investigated in the following analysis.

\subsection{Coherent Structure Evolution}

The experimental research of D'Ovidio and Coats (2013), and counterpart simulation work by McMullan et al. (2015) discovered that the mechanism of growth of the mixing layer differs in the pre- and post-transition regions. In the pre-transition region, the mixing layer grows through pairing-type interactions that were documented by Winant and Browand (Winant and Browand 1974). In the post-transition region, however, the coherent structures grow in a continuous, linear manner, with the interactions between coherent structures contributing nothing to the overall growth of the mixing layer. The growth of the pre-transition mixing layer through the pairing of primary vortices has been demonstrated in both experiments and numerical simulations, and is not repeated here. The focus in this section is on the evolution of the post-transition structures.

The coherent structures in the post-transition region are analysed from sequences of up to nine hundred frames of flow visualisation outputs. In these images the polar points of the coherent structures are tracked from frame to frame, and the resultant structure diameter is plotted as a function of streamwise distance. The evolution of several typical structures in each simulation is plotted in Figure 9a - 9c. In Case D1, the structures begin their lives with a given diameter. As they proceed downstream, the diameter of the structure does not vary significantly, and all posttransition coherent structures in Case D1 display no evidence of continuous linear growth. In Case D2 (Figure 9b), the evolution of the coherent structures undergoes a marked change at $x / \theta_{i} \approx 760$ - upstream of this location, the structures do show evidence of continuous growth, but downstream of this location the structures revert to maintaining a fixed diameter throughout their lifetimes. The behaviour of the structures in Cases D1 and D2 can be linked to the effect of spanwise wavelength confinement of the mixing layer. Confinement occurs when the aspect ratio of the flow attains a value of $A=10$. In Case D1, this criterion is satisfied at $x / \theta_{i}=360$ - a streamwise location which roughly corresponds to the mean transition location of the flow. All structures which form beyond the transition will be confined by the spanwise domain, and continuous linear growth is inhibited. In Case D2, confinement occurs at $x / \theta_{i}=760$ - the location where the continuous linear growth of coherent structures in the flow ceases. Given that the coherent structures occupy the entire visual thickness of the layer, and that visual thickness of the flow, $\delta_{v i z} \approx 10 \theta$, it can be inferred that the spanwise wavelength of the coherent structures is $\Lambda_{z} \approx \delta_{v i z}$. The criteria for confinement can then be stated as the flow being adversely affected by the spanwise domain when the local visual thickness approaches the spanwise domain extent.

The tracks of the individual structures in Case D3 shown in Figure 9c demonstrate that the structures in this simulation undergo continuous linear growth throughout the post-transition region of the flow. The structures occupy the full visual thickness of the mixing layer, with cycle-to-cycle variability in the location of the virtual origin accounting for the variability in the structure diameter when compared to the mean visual thickness. The mean flow statistics presented above have shown that Case D3 displays no evidence of flow confinement, and this is because the post-transition structures are free to grow in a continuous linear manner throughout the entire post-transition extent of the simulated mixing layer.

The flow visualisation images in Figures $4 \& 5$ show that both the size and spacing of the coherent structures increase with streamwise distance. This implies that some form of interaction takes place between the structures as they proceed downstream. The growth tracks of coherent structures participating in a typical merging interaction in case D1 is shown in Figure 10a. Both parent 
structures maintain a reasonably constant diameter prior to the interaction, and the interaction results in a stepwise growth in the diameter of the daughter structure. As the daughter structure proceeds downstream, it also maintains a roughly constant diameter. This type of interaction has been commonly observed in the pre-transition region (Winant and Browand (1974); D'Ovidio and Coats (2013)), but is at odds with the continuous growth observed in high Reynolds number mixing layers (D'Ovidio and Coats 2013). The growth tracks of structures undertaking a merging interaction in Case D3 are shown in Figure 10b. Both parent structures undergo continuous linear growth up to the point of merging, and the daughter structure that emerges also undergoes continuous growth. From the tracks it is apparent that, if the interaction had not occurred, either parent structure would have occupied the same visual thickness of the flow as the daughter structure. For the unconfined post-transition flow in simulation D3, the interactions contribute nothing to the overall growth of the mixing layer, and continuous linear growth is the dominant growth mechanism. Prior to confinement, the interactions between structures in Case D2 are of the form observed in Case D3. Following confinement in Case D2, the interactions switch to the type observed in Case D1.

\section{Conclusions}

Large Eddy Simulations of spatially developing mixing layers have been performed, for the purposes of clarifying the influence of the spanwise domain extent, and therefore the maximum spanwise wavelength that can be attained by the mixing layer, on the computed flow field. The boundary conditions represent an idealised form of the experimental flow. The mixing layer flow development becomes confined when the ratio of spanwise domain to momentum thickness reaches a value of ten. Upon confinement, the momentum thickness growth rate of the mixing layer is reduced, and the peak streamwise velocity fluctuation level drops when compared to both experimental data, and unconfined LES data. Interrogation of flow visualisation images reveals that, upon confinement, the post-transition coherent structures maintain a roughly constant diameter, and growth of the mixing layer occurs through interactions between structures. For an unconfined post-transition mixing layer, the coherent structures undergo continuous linear growth, with interactions between structures producing no additional growth. The results presented here demonstrate the importance in specifying an adequate computational domain for the simulation of the mixing layer. In spite of its classification as a two-dimensional flow, the transient evolution of the simulated turbulent coherent structures is inherently three-dimensional, and an inadequate specification of the spanwise domain extent will result in erroneous predictions of the flow.

\section{Acknowledgement}

The simulations presented in this paper were performed using ALICE, the University of Leicester High Performance Computing facility.

\section{References}

Attili, A., and Bisetti, B. 2012. "Statistics and scaling of turbulence in a spatially developing mixing layer at $\operatorname{Re}_{\lambda}=250$." Physics of Fluids, Vol. 24, doi: 10.1063/1.2696302

Batt, R. G. 1975. "Some measurements on the effect of tripping the two-dimensional shear layer." $A I A A$ Journal, Vol. 13, pp. 245-247.

Bell, J. H., and Mehta, R. D. 1992. "Measurements of the streamwise vortical structure in a plane mixing layer." Journal of Fluid Mechanics, Vol. 239, pp. 213-248.

Bernal, L. P., and Roshko, A. 1986. "Streamwise vortex structure in plane mixing layers." Journal of Fluid Mechanics, Vol. 170, pp. 499-525. 
Bernard, P. S., 2008. "Grid-free simulation of the spatially growing mixing layer." AIAA Journal, Vol. 46, pp. $1725-1737$.

Biancofiore, L. 2014. "Crossover between two- and three-dimensional turbulence in spatial mixing layers." Journal of Fluid Mechanics, Vol. 745, pp. 164-179.

Bogey, C., and Baiily, C. 2010. "Influence of nozzle-exit boundary-layer conditions on the flow and acoustic fields of initially laminar jets." Journal of Fluid Mechanics, Vol. 663, pp. 507-538.

Browand, F. K, and Latigo, B. O. 1979. "Growth of the two-dimensional mixing layer from a turbulent and nonturbulent boundary layer." Physics of Fluids, Vol. 22, pp. 1011-1019.

Brown G. L.,and Roshko A.. 1974. "On density effects and large structure in turbulent mixing layers." Journal of Fluid Mechanics, Vol. 64, pp. 755-816.

Dimotakis P. E. 200. "The mixing transition in turbulent flows." Journal of Fluid Mechanics, Vol. 409, pp. 69-98.

D'Ovidio, A., and Coats, C. M. 2013. "Coherent-structure evolution in turbulent mixing layers. Part 1: Experimental evidence." Journal of Fluid Mechanics, Vol. 737, 2013, pp. 466-498.

Gao, S., and Voke, P. 1995. "Large-eddy simulation of turbulent heat transport in enclosed impinging jets." International Journal of Heat and Fluid Flow, Vol. 16, pp.349-356.

Hadjadj, A., and Kudryavstev, A. 2005. "Computation and flow visualisation in high-speed aerodynamics." Journal of Turbulence, Vol. 6, doi: 10.1080/14685240500209775

Haller, G. 2005. "An objective definition of a vortex." Journal of Fluid Mechanics, Vol. 525, pp. 1-26.

Hernan M. A., and Jimenez J. 1982. "Computer analysis of a high-speed film of a plane turbulent mixing layer." Journal of Fluid Mechanics, Vol. 119, pp. 323-345.

Huang L-S, and Ho C-M. 1990. "Small scale transition in a plane mixing layer." Journal of Fluid Mechanics, Vol 210, pp. 475-500.

Jimenez, J. 1983. "A spanwise structure in the plane shear layer." Journal of Fluid Mechanics, Vol. 132, pp. 319-336.

Karasso P. S., and Mungal M. G. 1996. "Scalar mixing and reaction in plane liquid shear layers." Journal of Fluid Mechanics, Vol. 323, pp. 23-63.

Konrad J. H. 1976. "An experimental investigation of mixing in two-dimensional shear flows with applications to diffusion limited chemical reactions." PhD thesis, California Institute of Technology.

Lasheras, J. C., Choi, J. S., and Maxworthy, T. 1986. "On the origin and evolution of streamwise vortical structures in a plane, free shear-layer." Journal of Fluid Mechanics, Vol. 172, pp. 231-258

Liepmann H. W., and Laufer J. 1947. "Investigation of free turbulence mixing." NACA tech. note No. $12 \%$.

Lesieur, M., Comte, P., Lamballais, E., Mtais, O., and Silvestrini, G. 1997. "Large-eddy simulations of shear flows." Journal of Engineering Mathematics, Vol. 32, pp. 195-215.

McMullan, W. A., Coats, C. M., and Gao, S. 2011. "Analysis of the variable density mixing layer using Large Eddy Simulation." 41st AIAA Fluid Dynamics Conference and Exhibit, Honolulu, HI. AIAA-2011-3424.

McMullan W. A., Gao S., and Coats C. M. 2007. "A comparative study of inflow conditions for two- and three-dimensional spatially developing mixing layers using Large Eddy Simulation." International Journal of Numerical Methods in Fluids, Vol. 55, pp. 589-610.

McMullan W. A., Gao S., and Coats C. M. 2009. "The effect of inflow conditions on the transition to turbulence in Large Eddy Simulations of spatially developing mixing layers." International Journal of Heat and Fluid Flow, Vol. 30, pp. 1054-1066.

McMullan, W. A., Coats, C. M., and Gao, S. 2010. "Investigation of coherent structures in turbulent mixing layers using Large Eddy Simulation." 48th AIAA Aerospace Sciences Meeting, Orlando, FL. AIAA-20101291.

McMullan W. A., Gao S., and Coats C. M. 2015. "Organised large structure in the post-transition mixing layer. Part 2. Large Eddy Simulation." Journal of Fluid Mechanics, Vol 762, pp. 302-343.

McMullan, W. A., and Page G. J. 2011. "Large eddy simulation of a compressor cascade and the influence of spanwise domain." Proceedings of the IMechE, Part A: Journal of Power and Energy, Vol. 225, pp. 817-831.

Moser R. D., and Rogers M. M. 1991. "Mixing transition and the cascade to small scales in a plane mixing layer." Physics of Fluids A, Vol. 3 pp. 1128-1134.

Moser R. D., and Rogers M. M. 1993. "The three-dimensional evolution of a plane mixing layer: pairing and transition to turbulence." Journal of Fluid Mechanics, Vol. 247, pp. 275-320.

Nicoud, F., and Ducros, F. 1999. "Subgrid-scale stress modelling based on the square of the velocity gradient tensor." Flow, Turbulence and Combustion, Vol. 62, pp. 183-200. 
Pierrehumbert, R. T., and Widnall, S. E. 1982. "The two- and three-dimensional instabilities of a spatially periodic shear layer." Journal of Fluid Mechanics, Vol. 114, pp. 59-82.

Quirk, J.J. 1994. "A contribution to the great Riemann solver debate." International Journal of Numerical Methods in Fluids, Vol. 18, pp. 555-574.

Rogers M. M., and Moser R. D. 1992. "The three-dimensional evolution of a plane mixing layer: the KelvinHelmholtz rollup." Journal of Fluid Mechanics, Vol. 243, pp. 183-226.

Slessor, M. D., Bond, C. L., and Dimotakis, P. E. 1998. "Turbulent shear-layer mixing at high Reynolds numbers: effects of inflow conditions." Journal of Fluid Mechanics, Vol. 375, pp. 115-138.

Soteriou, M. C., and Ghoniem, A. F. 1998. "On the effects of the inlet boundary condition on the mixing and burning in reacting shear flows." Combustion and Flame, Vol. 112, pp. 404-417.

Soteriou, M. and Yang, X. 1999. "Inlet condition effects on particle dispersion in a shear layer." Combustion Science and Technology, Vol. 148, pp. 59-92.

Wang, P., Frolich, J., Michelassi, V., and Rodi, W. 2008. "Large-eddy simulation of variable-density turbulent axisymmetric jets." International Journal of Heat and Fluid Flow, Vol. 29, pp. 654-664.

Voke, P., and Potamitis, S. G. 1994. "Numerical simulation of a low-Reynolds-number turbulent wake behind a flat plate." International Journal of Numerical Methods in Fluids, Vol. 19, pp. 377-393.

Wang Y., Tanahashi M., and Miyauchi T. 2007. "Coherent fine scale eddies in turbulence transition of spatially-developing mixing layer." International Journal of Heat and Fluid Flow, Vol. 28, pp. 1280-1290.

Winant C. D., and Browand F. K. 1974. "Vortex Pairing: The mechanism of turbulent mixing layer growth at moderate Reynolds numbers." Journal of Fluid. Mechanics, Vol 63, pp. 237-255.

Wygnanski I., and Fiedler H. E. 1970. "The two-dimensional mixing region." Journal of Fluid Mechanics, Vol. 41, pp. 327-361.

Yang W. B., Zhang H. Q., Chan C. K., Lau K. S., and Lin W. Y. 2004. "Investigation of plane mixing layer using large eddy simulation." Computational Mechanics, Vol. 34, pp. 423-429.

Zhou X. Y., and Pereira J. C. F. 2000. "Large Eddy Simulation (2D) of a Reacting Plane Mixing Layer Using Filtered Density Function Closure." Flow, Turbulence and Combustion, Vol. 64, pp. 279-300. 
Table 1. Flow Parameters.

\begin{tabular}{ccccc}
$U_{1}\left(\mathrm{~ms}^{-1}\right)$ & $\theta_{1}(\mathrm{~mm})$ & $U_{2}\left(\mathrm{~ms}^{-1}\right)$ & $\theta_{2}(\mathrm{~mm})$ & $\mathrm{R}$ \\
\hline 25.6 & 0.457 & 5.2 & 0.86 & 0.66 \\
\hline
\end{tabular}

Table 2. Computational Domains.

\begin{tabular}{lcccccc} 
Case & $L_{x} / \theta_{i}$ & $L_{y} / \theta_{i}$ & $L_{z} / \theta_{i}$ & $N_{x}$ & $N_{y}$ & $N_{z}$ \\
\hline D1 & 1630 & 1326 & 98 & 768 & 256 & 64 \\
D2 & 1630 & 1326 & 196 & 768 & 256 & 128 \\
D3 & 1630 & 1326 & 392 & 768 & 256 & 256 \\
\hline ins. & & & & & &
\end{tabular}

Table 3. Growth rate constants.

\begin{tabular}{lcc} 
Case & $k_{1}$ & $k_{2}$ \\
\hline D1 & 0.031 & 0.362 \\
D2 & $0.0318,0.0305$ & 0.32 \\
D3 & 0.0318 & 0.319 \\
Expt & 0.033 & - \\
\hline
\end{tabular}

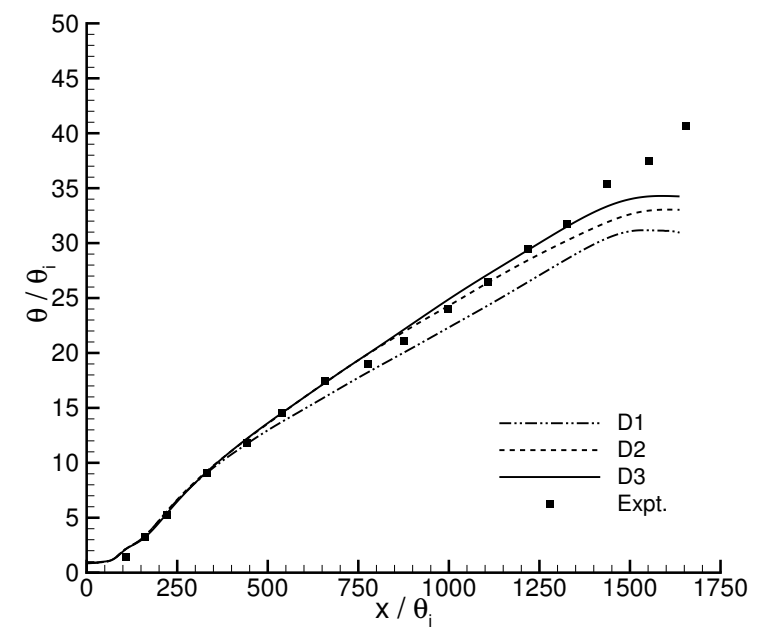

(a) Momentum thickness.

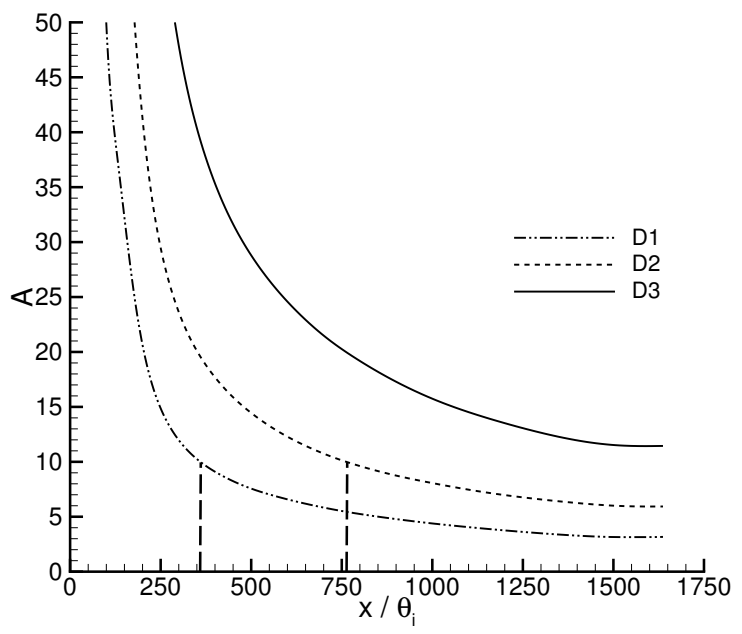

(b) Aspect ratio.

Figure 1. Integral scale variations of the simulations. 


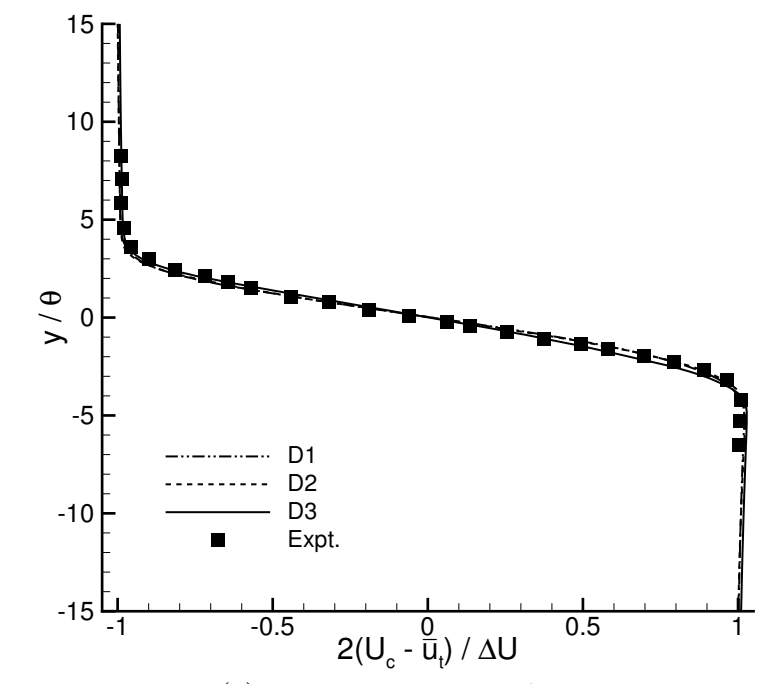

(a) Mean streamwise velocity.

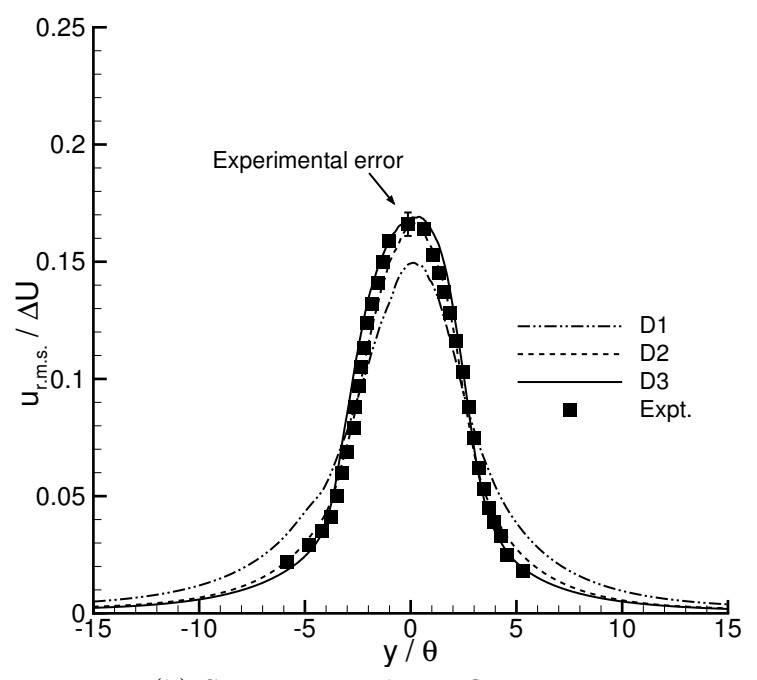

(b) Streamwise velocity fluctuations.

Figure 2. Flow statistics gathered at $x / \theta_{i}=1000$.

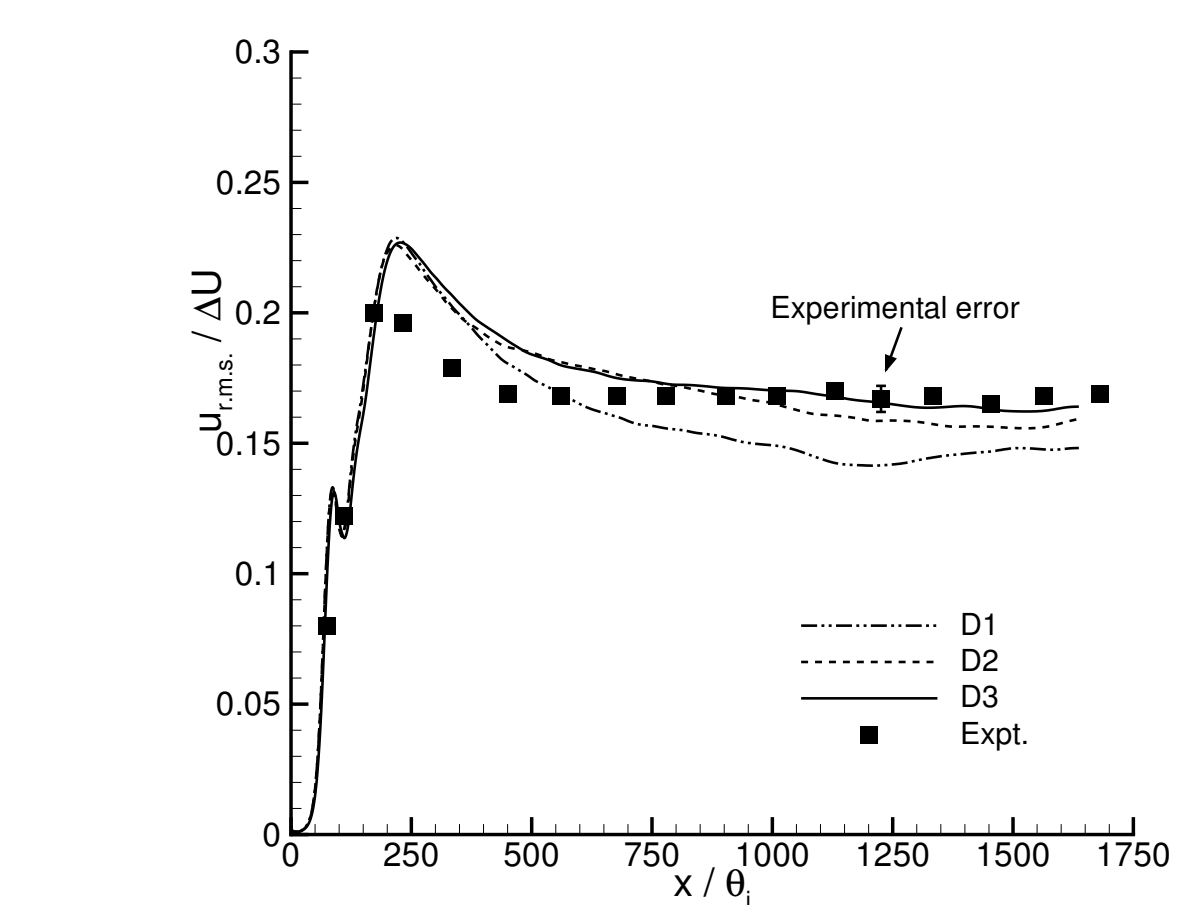

Figure 3. Variation of maximum streamwise velocity fluctuation with streamwise distance. 


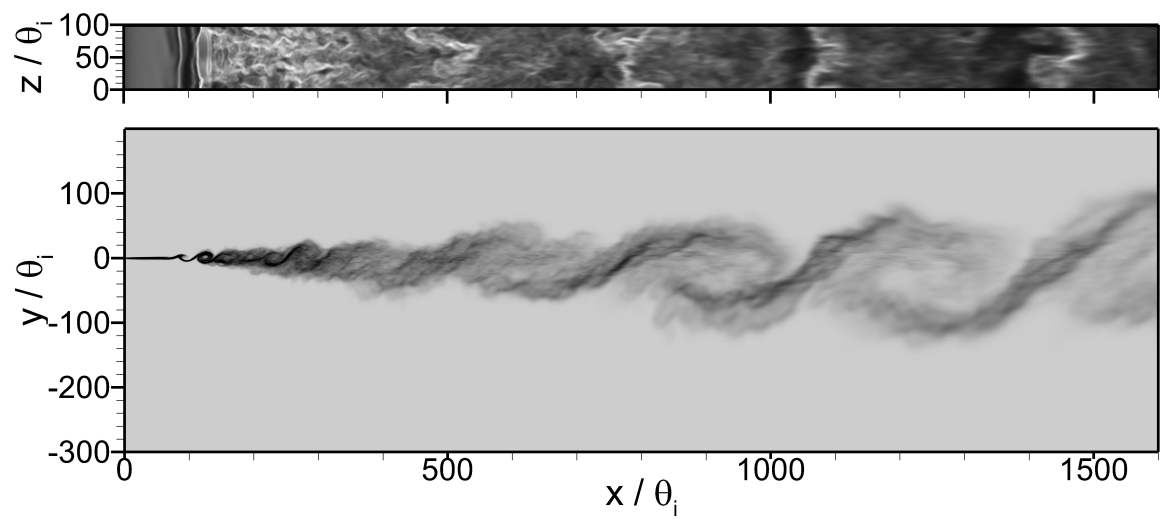

Figure 4. Instantaneous numerical schlieren of case D1. Top image, plan view. Bottom image, side view.
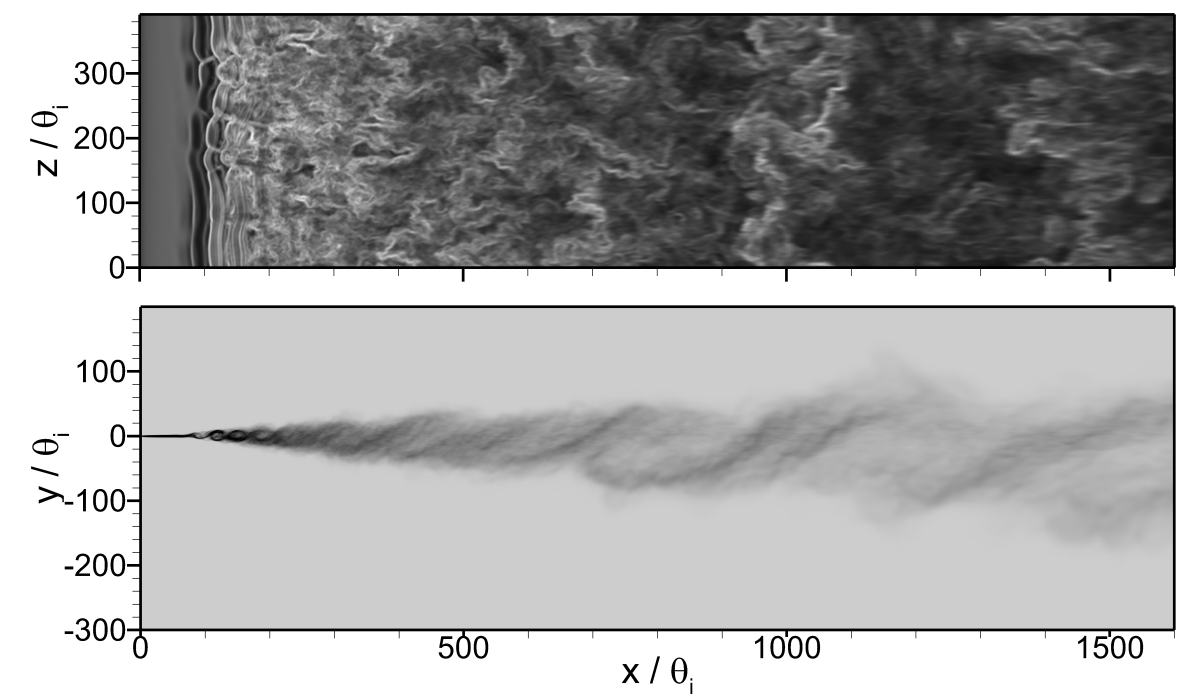

Figure 5. Instantaneous numerical schlieren of case D3. Top image, plan view. Bottom image, side view.

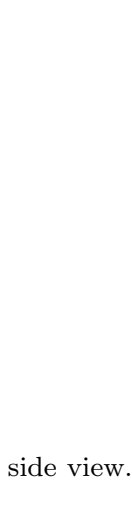

$$
\text { . }
$$

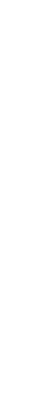

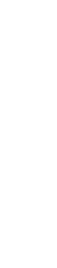


$\bar{\xi}: \quad 0.010 .150 .290 .430 .570 .710 .850 .99$

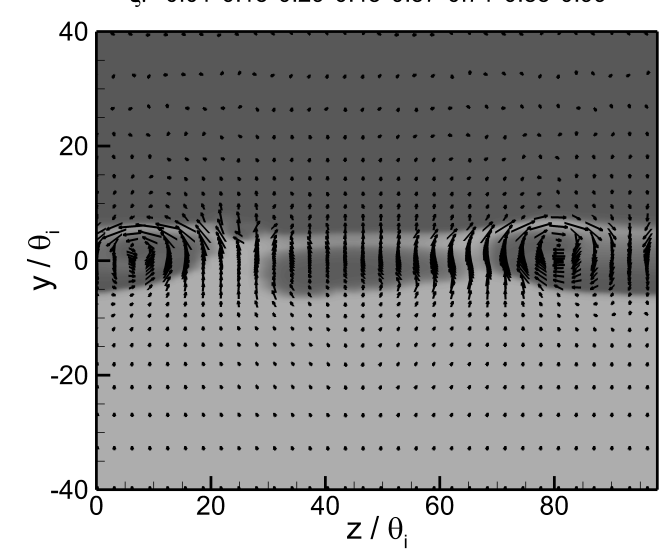

(a) Case D1.

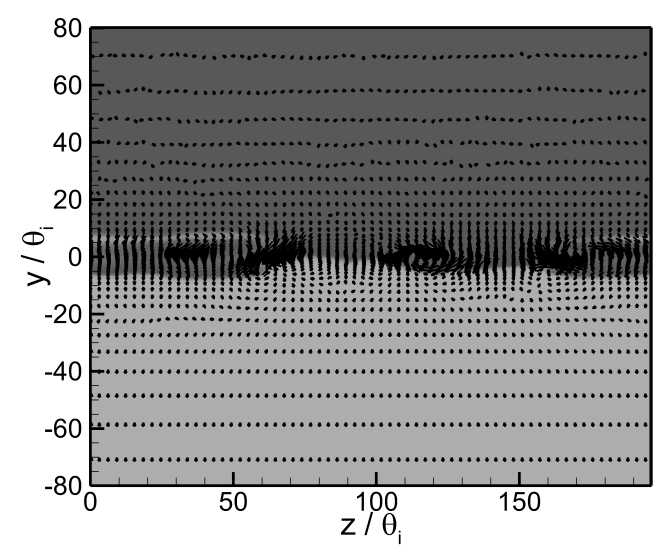

(b) Case D2.

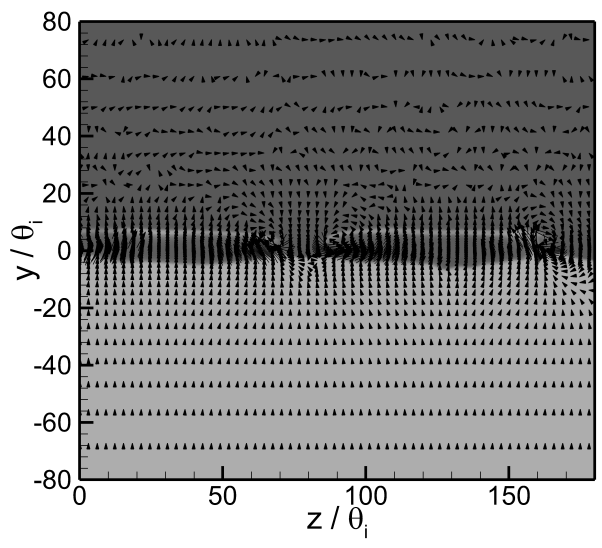

(c) Case D3.

Figure 6. $y-z$ cuts through a coherent structure at $x / \theta_{i}=108$. Only half of the computational domain is shown in Case D3 for clarity. Note the change in axis lengths between figures. 


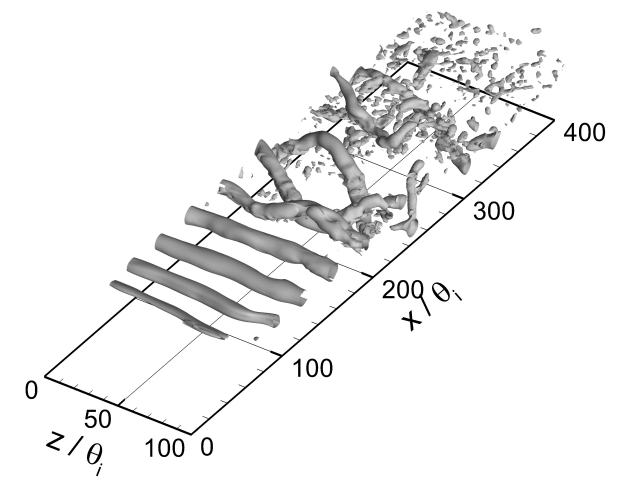

(a) Case D1.

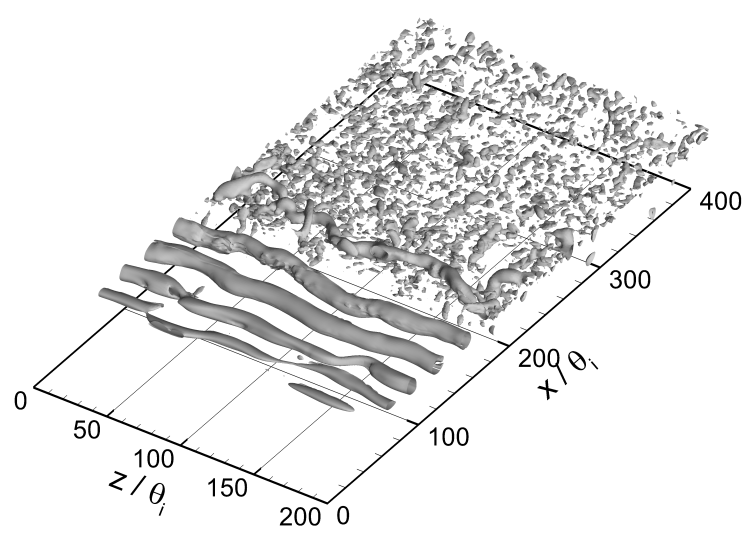

(b) Case D2.

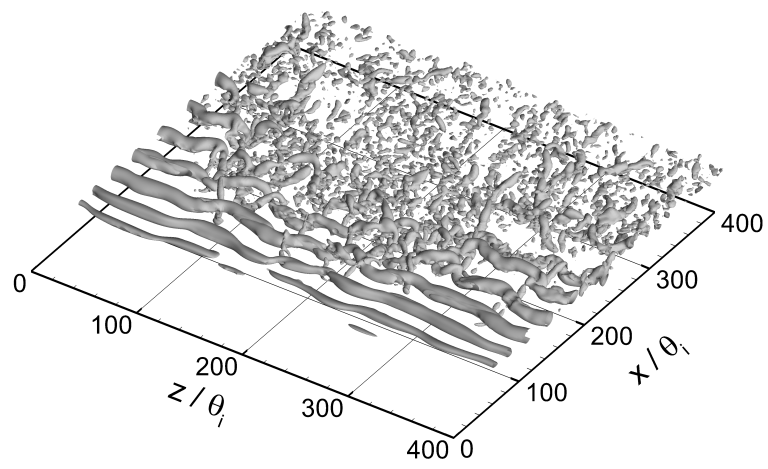

(c) Case D3.

Figure 7. Q-criterion iso-surfaces in the pre-transition region of each simulation.

$\bar{\xi}: \quad 0.010 .150 .290 .430 .570 .710 .850 .99$

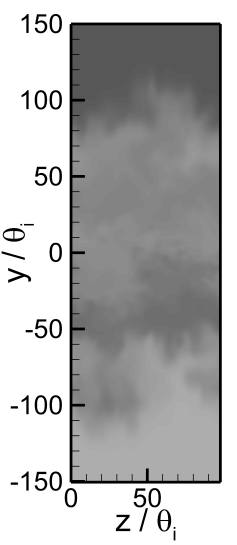

(a) Case D1.

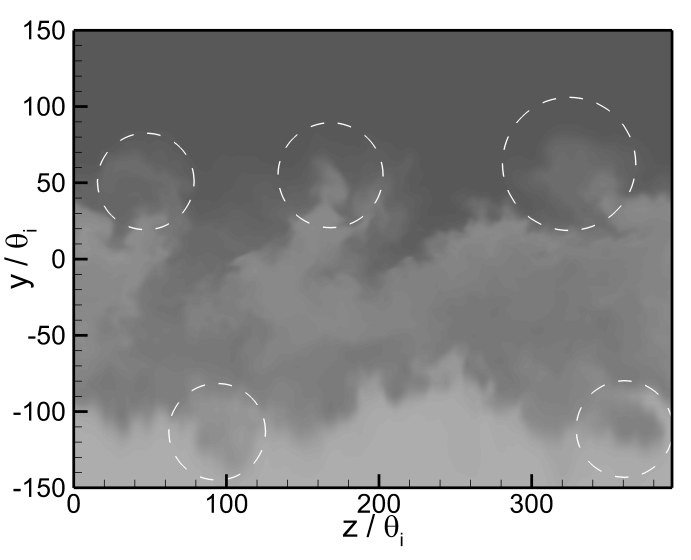

(b) Case D3.

Figure 8. $y-z$ cuts through a coherent structure at $x / \theta_{i}=1304$. 


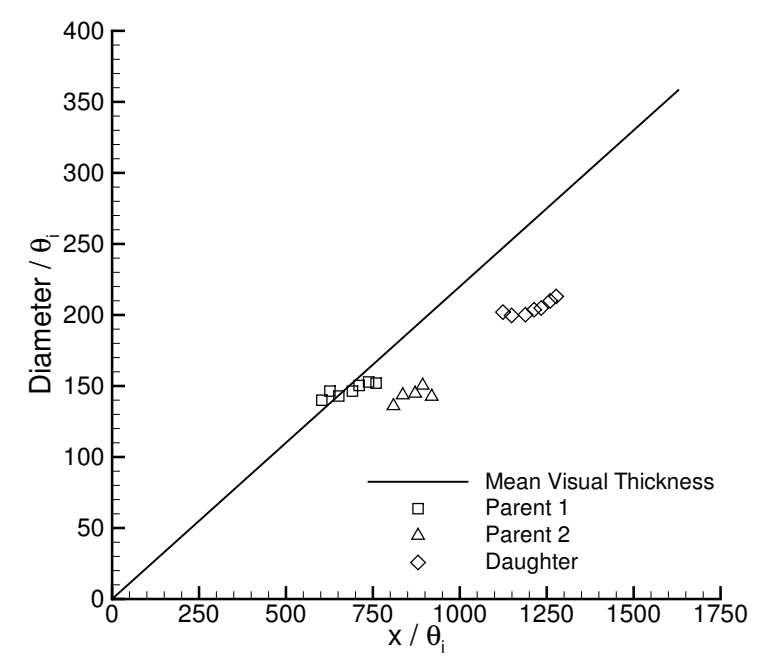

(a) Case D1. indicate a continuously growing structure.

rar

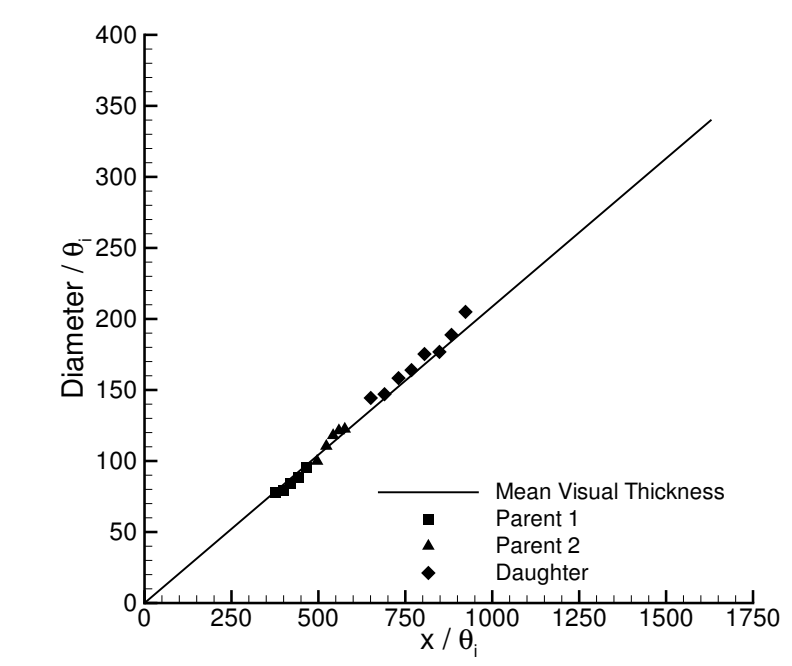

(b) Case D3.

Figure 10. Variation of participating structure diameter during a merging interaction in the post-transition flow. Filled symbols 